\title{
Molecular Fluorophores Self-Organize into C-dot Seeds and Incorporate into C-dot Structures
}

\author{
Michal Langer", Markéta Paloncýová", Miroslav Medved', Michal Otyepka*
}

Regional Centre of Advanced Technologies and Materials, Department of Physical Chemistry,

Faculty of Science, Palacký University Olomouc, 17. listopadu 12, 77146 Olomouc, Czech

Republic

\section{Corresponding Author}

*michal.otyepka@upol.cz 
ABSTRACT Various molecular fluorophores have been identified to be present during carbon-dot (C-dot) syntheses. However, the organization of such fluorophores in C-dots is still unknown. We study the self-assembly of 5-oxo-1,2,3,5-tetrahydroimidazo-[1,2- $\alpha]$-pyridine-7-carboxylic acid (IPCA), a molecular fluorophore present during the synthesis of C-dots from citric acid and ethylenediamine. Both forms of IPCA (neutral and anionic) show a tendency to self-assemble into stacked systems, forming seeds of C-dots during their synthesis. IPCA also interacts with graphitic C-dot building blocks, and fragments easily incorporates into their structures via $\pi-\pi$ stacking. Both IPCA forms are able to create ad-layers internally stabilized by an extensive hydrogen bonding network, with an arrangement of layers similar to that in ordinary graphitic C-dots. The results show the tendency of molecular fluorophores to form organized stacked seeds of C-dots and incorporate into C-dot structures. Such noncovalent structures can be further covalently interlinked via the carbonization process during C-dot growth.

\section{TOC GRAPHICS}




Although carbon dots (C-dots) were only first discovered in 2004, they have rapidly become one of the most extensively studied carbon-based nanomaterials. Their facile synthesis, stability, biocompatibility and extensive luminescence properties ${ }^{1,2}$ make C-dots a promising material for optoelectronics, ${ }^{3,4}$ theranostics,${ }^{5,6}$ drug delivery, ${ }^{7}$ sensing applications ${ }^{8}$ and bioimaging. ${ }^{9}$ A C-dot is a quasi-spherical 0D nanomaterial (usually smaller than $10 \mathrm{~nm}$ ) which consists of a graphite-like core of graphene sheets stacked on each other and an outer shell bearing oxygen- or nitrogencontaining functional groups. ${ }^{2,10-12}$ The detailed structure of C-dots is currently under intensive debate owing to their complexity and non-uniform nature. C-dot photoluminescence (PL) is also complex, but some common features can be identified. In particular, it is possible to distinguish among core, surface and molecular states ${ }^{13-16}$ contributing to C-dot PL..$^{17,18}$

Recent discoveries have shown that molecular fluorophores can also be integrated in the structure of C-dots, ${ }^{19}$ significantly affecting the PL properties.${ }^{18,20-25}$ Song et al..${ }^{17}$ demonstrated the presence of a fluorescent molecule, i.e., 5-oxo-1,2,3,5-tetrahydroimidazo-[1,2- $\alpha]$-pyridine-7carboxylic acid (IPCA), in solution when preparing C-dots via a bottom-up synthesis from citric acid and ethylenediamine and showed that it significantly increased the fluorescent quantum yield and shifted the absorption/emission spectra. As recently reviewed ${ }^{26}$ aggregation of several fluorescent molecules in solvent has been observed experimentally, e.g., 5-oxo-3,5-dihydro-2Hthiazolo[3,2-a]pyridine-7-carboxylic acid (TPCA), 5-oxo-3,5-dihydro-2H-thiazolo[3,2a]pyridine-3,7-dicarboxylic acid (TPDCA) ${ }^{27,28}$ and hydroxymethylfurfural (HMF).${ }^{19}$ Thus, it has been suggested that these aggregates may affect the overall fluorescence properties of a C-dot solution. ${ }^{29-32}$ These findings are consistent with the fact that planar conjugated materials tend to form aggregates in solutions/suspensions. ${ }^{33-35}$ Self-assemblies of polyaromatic hydrocarbons (PAHs) used as building blocks display PL properties resembling those of C-dots, and the 
properties of C-dots can be tuned by changing the composition of individual PAH building blocks. ${ }^{36}$ Self-assemblies of luminescent molecules have been suggested to play a significant role in the C-dot formation process ${ }^{16}$ and contribute to C-dot PL. However, the precise structural features of such assemblies are still unknown and particularly challenging to determine by experimental methods owing to their complexity and expected inherent dynamics. Understanding the structural features of such assemblies may help to better understand the processes of C-dot formation and origins of C-dot PL enabling rational design of C-dots with tailored properties and facilitate theoretical studies dealing with the PL properties of C-dots. ${ }^{37-42}$

In this work, we employed classical all-atom molecular dynamics (MD) simulations in explicit solvent to analyze the self-assembly process of the molecular fluorophore IPCA and the structural features of such self-assemblies in water. We also analyzed interactions of IPCA molecules with graphitic C-dots fragments. We focused on the self-assembly of IPCA anions (Scheme S1 in Supporting Information, estimated $\mathrm{p} K_{\mathrm{a}}$ of IPCA COOH group is 2.9 according to MarvinSketch 18.17. $0^{43}$ ) as the dominant protonation form close to neutral $\mathrm{pH}$. Results for the neutral IPCA form, i.e., a dominant form in an acidic environment, are shown in the Supporting Information. MD simulations of five systems were carried out (Table 1): (1) self-assembly of IPCAs, (2) interaction of IPCAs with a C-dot fragment, (3) interactions of IPCAs with a spherical C-dot model, and (4 and 5) self-assembly of edge functionalized PAHs ${ }^{44}$ without (4)/with (5) IPCAs in water. Systems (4) and (5) were also studied with a set of PAH layers with smaller radius (see Table S1 for a full list of simulations and system details). Although we performed all simulations with three independent replicas, the results are presented for only one of them as they were very similar (if not stated otherwise). 
Table 1. Composition of simulation boxes with numbers of PAH layers, IPCA(-1) anions and water molecules. For each system, three independent replicas were performed ( $c f$. Table S1 in Supporting Information).

\begin{tabular}{|l|l|l|l|l|l|}
\hline System & C-dot & IPCA(-1) & Water & Box dimensions (nm) & Simulation length (ns) \\
\hline 1 & None* & 50 & 10894 & $7.4 \times 7.0 \times 6.7$ & 200 \\
\hline 2 & 4 layers & 20 & 7302 & $6.1 \times 6.1 \times 6.1$ & 200 \\
\hline 3 & 9 layers & 10 & 10753 & $7.4 \times 7.0 \times 6.7$ & 200 \\
\hline 4 & 9 layers & 0 & 8280 & $6.5 \times 6.5 \times 6.5$ & 200 \\
\hline 5 & 6 layers* & 15 & 8360 & $6.5 \times 6.5 \times 6.5$ & 200 \\
\hline
\end{tabular}

*These simulations were also performed at temperature $473 \mathrm{~K}$ and pressure 15.5 bar.

\section{Self-assembly of IPCAs in water}

MD simulations showed the spontaneous and rapid formation of IPCA stacked assemblies in a water environment. Initially, within the first few nanoseconds of the MD simulations, randomly distributed monomers of IPCA anions started to form small aggregates (containing 2-3 IPCA molecules) (Figs. 1a, 2a, b). The nanoclusters subsequently grew and self-assembled into longer fibrils interacting via $\pi-\pi$ stacking interactions (Figs. 1a, 2a, b, S1 in Supporting Information SI). The $\pi$-stacked layers were separated by $\sim 0.34 \mathrm{~nm}$, which corresponds to the separation of graphene layers in graphite and the distance between individual layers in C-dots. ${ }^{45,46}$ The negatively charged carboxylate groups of stacked IPCA molecules were oriented either in opposite directions or interacted via a counterion (salt-bridge, Figs. 1d-f, S1). Note that neutral IPCAs (see Figs. 2a, c, S2-S5) displayed even higher tendency to form long $\pi$-stacks stabilized by electrostatics and dispersion interaction as revealed by the symmetry-adapted perturbation theory (SAPT) ${ }^{47}$ analysis (Table S2 and Fig. S6), suggesting that $\mathrm{pH}$ may affect the structure of C-dot seeds during synthesis, as discussed in the literature. ${ }^{48}$ These findings also imply that longer $\pi$-stacks are destabilized by 
the unfavorable electrostatic repulsion of deprotonated carboxylic groups of IPCA anions. Overall, the MD simulations revealed a strong tendency of IPCA to form nanoclusters in an aqueous environment with a highly dynamic structure, as many reorganization events of stacks were observed during the entire simulation time (see movie in Supporting Information). Different reorganization schemes of stacks were followed in the simulations, e.g., dimers were formed either from two monomers or by the disintegration of larger stacks; and they either aggregated into longer stacks or dissolved back into two monomers. Therefore, the lifetime of particular stacks, e.g., dimers, was very diverse (median for several dimers in MD simulations was $\sim 8 \mathrm{~ns}$ ). The lifetime of larger stacks was even shorter $(0.5-2.4 \mathrm{~ns})$. The observed stacked nanoclusters of IPCA may form seeds for the formation of C-dots, e.g., during bottom-up thermal synthesis, as suggested in other experimental works. ${ }^{49}$ To corroborate this idea, we carried out MD simulations at higher temperature $(473 \mathrm{~K})$ and pressure $(15.5 \mathrm{bar})$ corresponding to synthetic conditions.$^{17}$ The tendency of IPCA clustering at these conditions with respect to ambient (300 K and 1 bar) conditions was reduced (Figs. 2d, e, f) due to a significant role of the entropic contribution to free energy of association (Tables S3). The stacked nanoclusters of IPCAs were also observed at the higher temperature and pressure conditions (Fig. S7). The layered structure of IPCA self-assemblies imprinted by $\pi-\pi$ stacking may explain the graphitic-like structure of C-dots. 




Figure 1. Snapshots taken from MD simulations (at 200 ns) of (a) System 1 (detailed in Table 1) showing self-assemblies of IPCA anions; (b) System 2 showing that IPCA anions can selfassemble and also stack on an accessible surface of a C-dot fragment (shown by darker colors), forming an ad-layer on the C-dot; (c) System 3 showing that IPCA can also interact with a spherical C-dot. (d, e, f) Insets taken from panel (a) showing examples of IPCA dimer, trimer and tetramer, respectively. On average, $\pi$-stacked layers were separated by $\sim 0.34 \mathrm{~nm}$ in all systems. Coloring scheme: cyan, black-carbon; red-oxygen; blue-nitrogen; white-hydrogens. Water and $\mathrm{Na}^{+}$are omitted for clarity. 

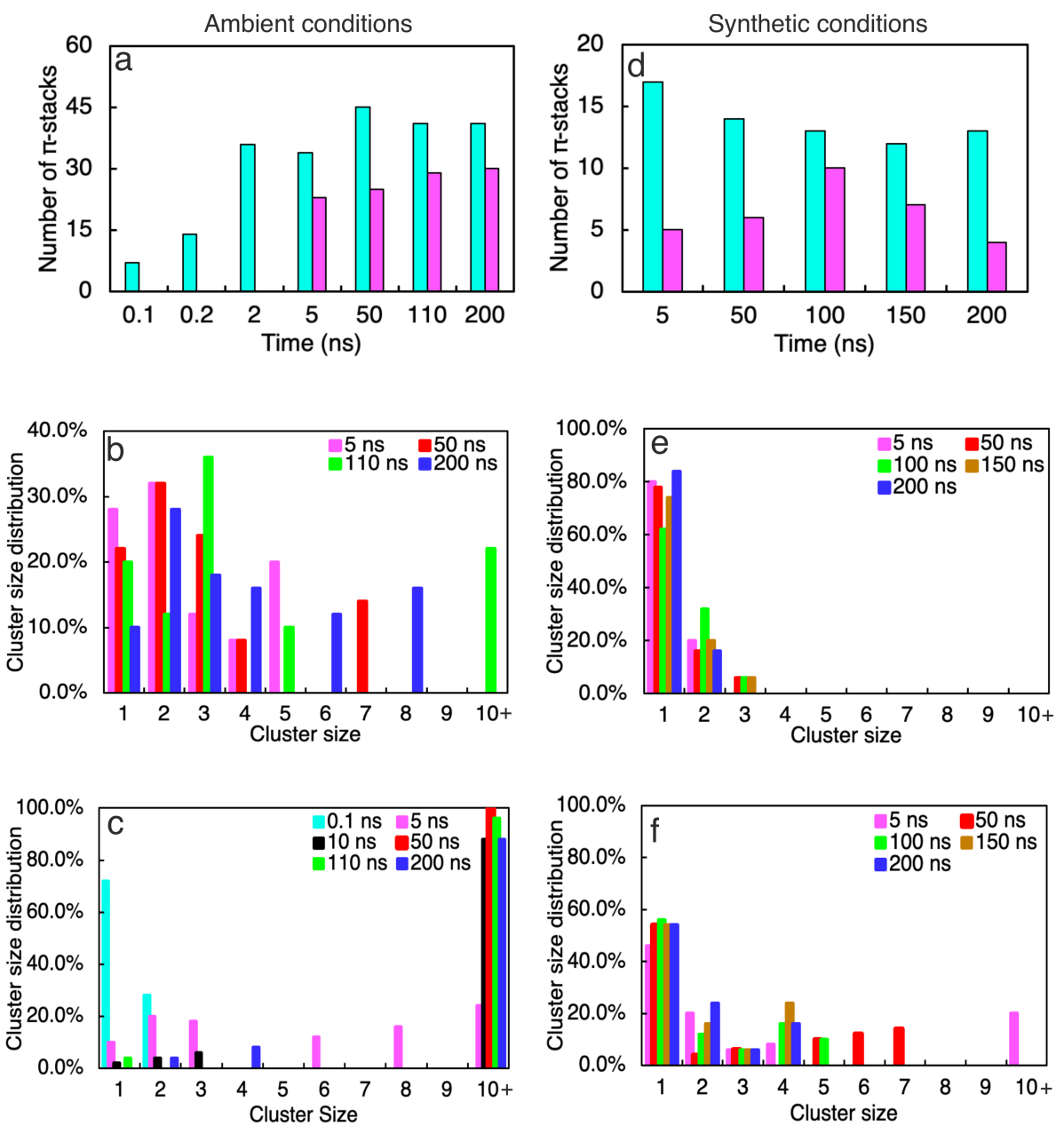

Figure 2. (a, d) Number of $\pi$-stacks of neutral (cyan) and deprotonated (magenta) IPCA molecules (system 1) collected at different times of MD simulations simulated at (a) $300 \mathrm{~K}$ and 1 bar; and (d) at $473 \mathrm{~K}$ and 15.5 bar, revealing the tendency of IPCA monomers to be self-assembled in water. Clustering analysis of (b) deprotonated IPCAs (300 K, 1 bar), (c) neutral IPCAs (300 K, 1 bar), (e) deprotonated IPCAs (473 K, 15.5 bar), (f) neutral IPCAs (473 K and 15.5 bar) analyzed for selected simulation times show the number of IPCAs that preferentially formed the clusters. Cluster size distribution refers to how many IPCAs of the total number are in the particular stack.

\section{Interaction of IPCAs with C-dot in water}

MD simulations were also used to investigate the affinity of IPCA anions to interact with a Cdot fragment, i.e., half-spherical C-dot. The graphitic C-dot fragment consisted of four layers of 
edge-functionalized PAHs (Fig. 1b). IPCA monomers interacted with the C-dot layers via $\pi$ stacking and preferentially stacked on layers with a solvent exposed surface (Figs. 1b, S8). IPCA anions created typically one or two or three ad-layers on the outer C-dot layer. The layer spacing was $\sim 0.34 \mathrm{~nm}$ (Figs. 3a, b), which is the same as for a (002) facet of graphitic fragments of C$\operatorname{dot}^{50-52}$ During the assembly process, molecules of IPCA also interacted with the C-dot fragment via $\mathrm{OH}-\mathrm{COO}(\mathrm{H})$ hydrogen bonds (HBs, Fig. S8), but such complexes were unstable and broke apart within a few ns. In the case of neutral IPCA molecules, more layers composed of exclusively IPCA molecules were formed (Fig. S9) with IPCAs connected by HBs. These layers were very similar to C-dot layers formed of PAHs (Fig. S9). Hence, the assembly process led to the formation of C-dots with graphitic structure that were hardly distinguishable from PAH C-dots in terms of their density profile (Fig. S9).

MD simulation of IPCA anions (and neutral molecules) with a fully spherical C-dot in solution (System 3) showed a similar stacking behavior and formation of temporary HBs with hydroxyl groups on the C-dot surface shell (Figs. 1c, S10-12). The lifetime of such HBs was $\sim 39$ ps, and they were repeatedly formed between IPCA and the C-dot or water molecules (analyzed by the intermittent HB correlation function using the gmx hbond tool for the first $100 \mathrm{~ns}$ of simulation time, as described by Luzar and Chandler ${ }^{53,54}$. For the sake of completeness, it should be noted that IPCA anions (and also neutral IPCA molecules) formed short-lived two- or three-membered stacks in solution (Fig. 1c). 

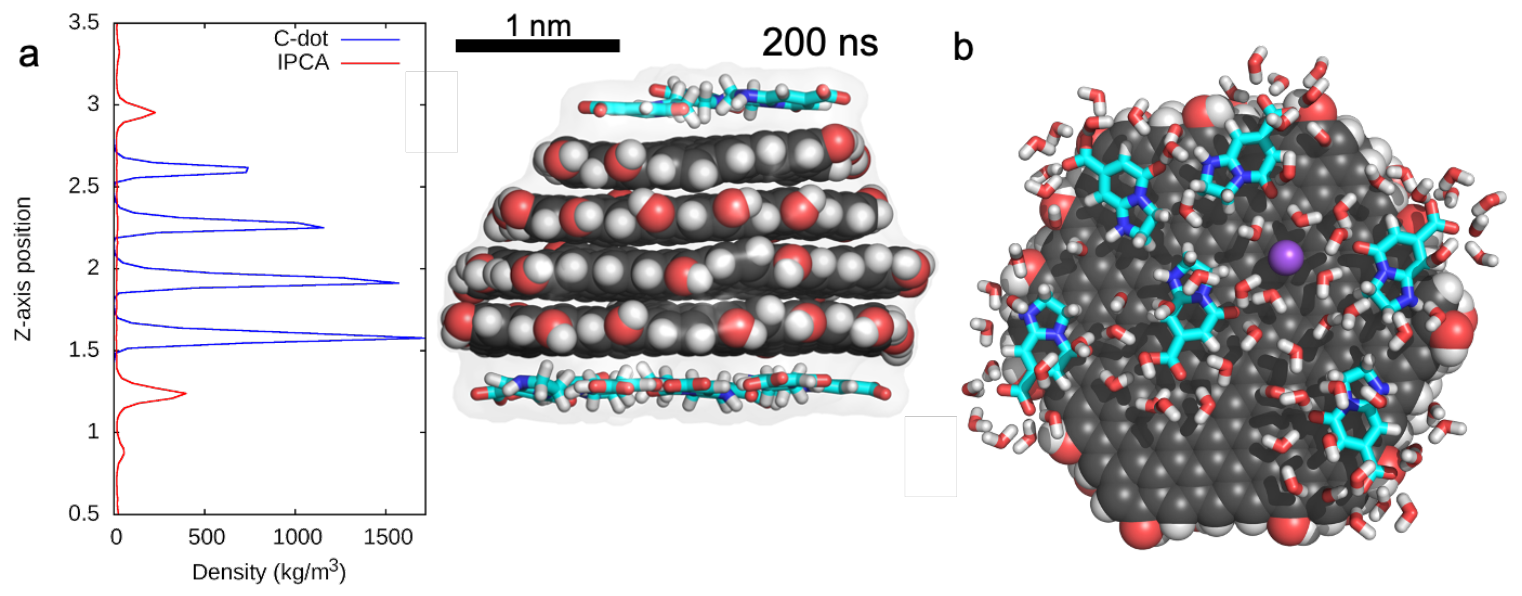

Figure 3. Panel a) shows density profile (left) of a C-dot fragment with IPCA anions showing the tendency of IPCA anions to stack on the outer layers of the C-dot fragment, forming nearly regular ad-layers with $0.34 \mathrm{~nm}$ interlayer spacing. Snapshot taken at $200 \mathrm{~ns}$ shows structure of the assembly of PAH layers (in black balls) and IPCAs (in cyan). Water, ions and dissolved IPCAs are omitted for clarity. Panel b) displays the same structure taken at $200 \mathrm{~ns}$ in a different view with an IPCA ad-layer displayed in detail with water molecules and $\mathrm{Na}^{+}$ion within $0.3 \mathrm{~nm}$ from IPCAs.

\section{Formation of C-dot from PAH building blocks in the presence of IPCA}

In addition to assembly of IPCAs on a C-dot fragment, we also investigated if and to what extent the presence of IPCA affects the process of C-dot self-assembly. We prepared randomly distributed hydroxyl edge-functionalized PAHs in water and analyzed their behavior with or without the presence of IPCA molecules. The simulations without IPCAs showed a tendency for PAHs to form graphitic C-dot structures stabilized by $\pi-\pi$ stacking (Figs. 4, S13-14). In contrast, if IPCA ions/molecules were present, ad-layers were formed via $\pi$-stacking interactions on top of PAH sheets (as seen in the MD simulations with a C-dot fragment). In addition, IPCA was able to incorporate/intercalate into the $\mathrm{C}$-dot structure, complementing/amending the PAH layers (Figs. 4, S15-19). In such a case, IPCA prefers to stay at the edge of the C-dot, with its carboxyl groups 
exposed to water (Fig. S20). Neutral IPCA molecules were able to form internal layers stabilized by HBs and sandwiched in-between PAHs layers (Figs. S19, S20). Although we observed that higher temperature $(473 \mathrm{~K})$ and pressure $(15.5 \mathrm{bar})$ conditions reduced the stacking ability of IPCAs in solution ( $c f$. Fig. 2), we did not detect any significant changes in MD simulations of PAH layers and IPCAs at these conditions (Fig. S21). This behavior can be attributed to higher affinities of IPCA(0) to PAH with respect to binding affinity between IPCA(0)s as evidenced by the potential of mean force (Fig. S22). All these findings suggest a mechanism by which molecular fluorophores may incorporate into a C-dot structure. ${ }^{17,18}$ During the carbonization process, these non-covalently bound systems may be further covalently linked, e.g., by dehydration reactions, to form final C-dot structures. ${ }^{18}$

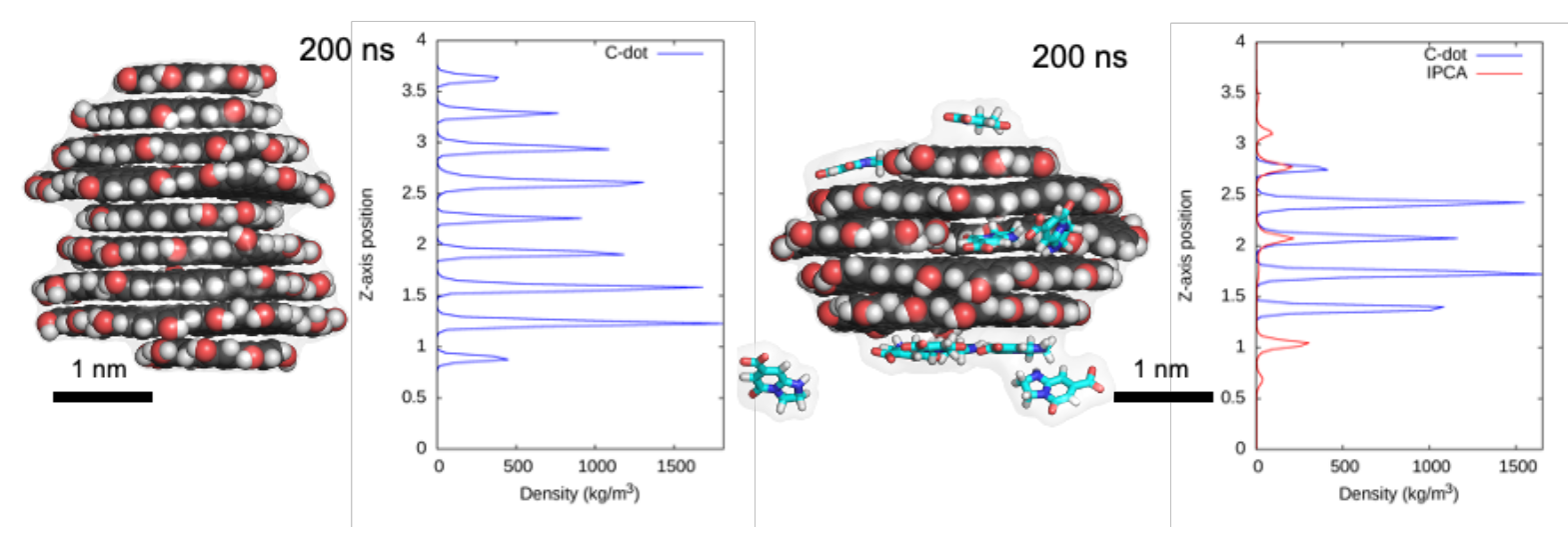

Figure 4. Final snapshots (taken at $200 \mathrm{~ns}$ ) of self-assembled C-dots composed of functionalized PAHs only (left: System $4 R_{\mathrm{g}}=1.16 \mathrm{~nm}$ ) and C-dots formed from functionalized PAHs in the presence of IPCA anions (right: System $5, R_{\mathrm{g}}=1.08 \mathrm{~nm}$ ) and their corresponding density profiles. PAH layers are represented by spheres (carbon - black, oxygen - red, hydrogen - white) and IPCA by sticks (carbons - cyan, nitrogen - blue). Water is omitted for clarity. 
In summary, all-atomic MD simulations revealed that IPCA molecules rapidly self-assembled in water to form $\pi-\pi$ stacked assemblies, which may serve as aggregation centers for C-dot formation, e.g., during the bottom-up synthesis of C-dots. Furthermore, if C-dot or a C-dot fragment was already present in the solution, IPCAs adsorbed on its surface, interacting mostly via $\pi$ - $\pi$ stacking on C-dot solvent exposed layers or alternatively through HBs with the surface functional groups. The complexes of C-dot with IPCA stabilized by HBs were relatively unstable and broke apart on a time scale of a few of nanoseconds. In contrast, the $\pi$ - $\pi$ stacked complexes were significantly more stable. IPCA was also capable of forming ad-layers on C-dot surfaces by creating a full layer of IPCAs internally stabilized by HBs. IPCAs together with functionalized PAHs were able to form C-dots with IPCA incorporated into their structures, preferably in solvent exposed (at least partially) poses. All these findings show that i) molecular fluorophores can spontaneously form C-dots seeds, ii) $\pi$ - $\pi$ stacking of molecular fluorophores in C-dot seeds facilitates the formation of graphitic C-dots, and iii) molecular fluorophores can readily incorporate into C-dot structures, where they can function as molecular fluorophores contributing to the PL properties of C-dots. ${ }^{17,18}$ 


\section{Computational Methods}

IPCA molecules were described by the GAFF force field. ${ }^{55}$ Parameters for the neutral form were taken from the literature ${ }^{56}$ (topology files are available in the Supporting Information) and parameters for the IPCA anion were derived by the same protocol ${ }^{56}$ (see Supporting Information). C-dots were described by the AMBER ff99 force field ${ }^{57}$ with refined parameters for aromatic carbon atoms ${ }^{58}$ Structures of C-dots were generated with carbon dot builder ${ }^{44}$ and PAH layers were functionalized on $30 \%$ of their edges by hydroxyl groups.

IPCA molecules were added randomly into a simulation box or around a C-dot/C-dot fragment. For Systems 4 and 5, we inserted first randomly the PAH layers and afterwards inserted IPCA molecules. Further, we solvated the systems with the SPC/E water model ${ }^{59}$ and neutralized them with $\mathrm{Na}^{+}$ions ${ }^{60}$ All systems were prepared and simulated as three replicas. After minimization with the steepest descent algorithm, MD simulations were performed under NpT conditions with a 2 fs time step, and bonds involving hydrogen were constrained using the LINCS algorithm. ${ }^{61}$ Owing to initial instability, Systems 4 and 5 were first subjected to $1 \mathrm{~ns}$ of heating, starting at 250 $\mathrm{K}$ and reaching $300 \mathrm{~K}$. In subsequent simulations, the temperature was kept at $300 \mathrm{~K}$ with the $\mathrm{V}$ rescale thermostat ${ }^{62}$ with a $0.1 \mathrm{ps}$ scaling constant. The isotropic Berendsen ${ }^{63}$ barostat was introduced to keep the pressure at 1 bar during the simulation, and the time constant for pressure relaxation was set to $2.0 \mathrm{ps}$. Electrostatic interactions were treated by means of the particle-mesh Ewald (PME) ${ }^{64}$ method with a real-space cutoff of $1.0 \mathrm{~nm}$; the same cutoff was applied for van der Waals interactions. Periodic boundary conditions were applied in all three dimensions. All MD simulations were performed in Gromacs 5.0. ${ }^{65}$ Further, MD simulations at temperature of $473 \mathrm{~K}$ and pressure 15.5 bar were performed in order to emulate experimental synthetic conditions ${ }^{17}$ when carbonization of C-dots occurs. 
Simulations were analyzed in terms of density profiles, the radius of gyration $\left(R_{\mathrm{g}}\right)$, number of hydrogen bonds (analyzed with GROMACS tools) and number of $\pi$-stacking interactions (evaluated with a home-written code,${ }^{66}$ analyzing the overlap of two IPCA planes geometrically, i.e., by a mathematical analysis of convex planar polygons for all ordered pairs of IPCAs with the stacking distance set to $0.3-0.4 \mathrm{~nm}$ ). Figures were rendered in PyMoL. ${ }^{67}$ 
ASSOCIATED CONTENT

AUTHOR INFORMATION

Corresponding Author

Michal Otyepka - Regional Centre of Advanced Technologies and Materials, Department of Physical Chemistry, Faculty of Science, Palacký University Olomouc, 17. listopadu 12, 77146 Olomouc, Czech Republic; Email: michal.otyepka@upol.cz

\section{Authors}

Michal Langer - Regional Centre of Advanced Technologies and Materials, Department of Physical Chemistry, Faculty of Science, Palacký University Olomouc, 17. listopadu 12, 77146 Olomouc, Czech Republic

Markéta Paloncýová - Regional Centre of Advanced Technologies and Materials, Faculty of Science, Palacký University Olomouc, 17. listopadu 12, 77146 Olomouc, Czech Republic

Miroslav Medved' - Regional Centre of Advanced Technologies and Materials, Faculty of Science, Palacký University Olomouc, 17. listopadu 12, 77146 Olomouc, Czech Republic

\section{Author contributions}

II M. L. and M. P. contributed equally.

\section{Notes}

The authors declare no competing financial interests.

\section{ACKNOWLEDGMENTS}

Support from the Internal Student Grant Agency of Palacký University in Olomouc, Czech Republic (IGA_PrF_2020_022), the ERC (project No. 683024 from the European Union's 
Horizon 2020), and the Ministry of Education, Youth and Sports (grant number: CZ.02.1.01/0.0/0.0/16-019/0000754). We would like to acknowledge Tomáš Kühr for providing the $\pi$-stacking code.

Supporting Information Available: Scheme S1: Structures of IPCA molecules with partial atomic charges; Table S1: Composition of simulation boxes; Figure S1: Evolution of IPCA anion self-assemblies; Figure S2: Cluster analysis of neutral IPCAs; Figure S3: Cluster analysis of neutral IPCAs in the long term; Figure S4: Evolution of neutral IPCA self-assemblies; Figure S5: Evolution of the number of hydrogen bonds between neutral IPCAs; Table S2: SAPT0 interaction energy decomposition for IPCA dimers; Figure S6: SAPT0 percentual contribution to the attractive interaction energy components; Table S3: MM and quantum mechanical calculations of stabilization and free energies of IPCA dimerization; Figure S7: Snapshots of MD simulations of IPCAs in solution performed under higher temperature and higher pressure; Figure S8: Evolution of the assembly process of IPCA anions with a C-dot fragment; Figure S9: Neutral IPCAs with a C-dot fragment; Figure S10: Evolution of the number of hydrogen bonds between IPCA anions and C-dot; Figure S11: Interaction of IPCA anions with a spherical C-dot; Figure S12: Interaction of neutral IPCAs with a spherical C-dot; Figure S13: Self-assembly of nine PAH layers; Figure S14: Self-assembly of five PAH layers; Figure S15: Self-assembly process of five PAH layers and five IPCA anions; Figure S16: Final structures of self-assembly of six PAH layers with IPCA anions; Figure S17: Assembly process of six PAH layers with IPCA anions; Figure S18: Selfassembly process of five PAH layers and five neutral IPCAs; Figure S19: Assembly process of six PAH layers with neutral IPCAs; Figure S20: Detail on hydroxyl-edge functionalization of system 5 and single layers' structures; Figure S21: Snapshots after 100 ns of unbiased simulations of PAH layers and IPCA molecules at high temperature and high pressure; Figure S22: Potential of mean 
force form MD; Summary of results of neutral IPCA; Topology of IPCA anion; Topology of neutral IPCA (PDF); Self-assembly of IPCA anions (MOV).

The Supporting Information is available free of charge.

\section{REFERENCES}

(1) Semeniuk, M.; Yi, Z.; Poursorkhabi, V.; Tjong, J.; Jaffer, S.; Lu, Z. H.; Sain, M. Future Perspectives and Review on Organic Carbon Dots in Electronic Applications. ACS Nano 2019, 13, 6224-6255.

(2) Lim, S. Y.; Shen, W.; Gao, Z. Carbon Quantum Dots and Their Applications. Chem. Soc. Rev. 2014, 44, 362-381.

(3) Yuan, T.; Meng, T.; He, P.; Shi, Y.; Li, Y.; Li, X.; Fan, L.; Yang, S. Carbon Quantum Dots: An Emerging Material for Optoelectronic Applications. J. Mater. Chem. C 2019, 7, 68206835.

(4) Guo, X.; Wang, C.-F.; Yu, Z.-Y.; Chen, L.; Chen, S. Facile Access to Versatile Fluorescent Carbon Dots toward Light-Emitting Diodes. Chem. Commun. 2012, 48, 2692-2694.

(5) Ge, J.; Jia, Q.; Liu, W.; Guo, L.; Liu, Q.; Lan, M.; Zhang, H.; Meng, X.; Wang, P. RedEmissive Carbon Dots for Fluorescent, Photoacoustic, and Thermal Theranostics in Living Mice. Adv. Mater. 2015, 27, 4169-4177.

(6) Du, J.; Xu, N.; Fan, J.; Sun, W.; Peng, X. Carbon Dots for In Vivo Bioimaging and Theranostics. Small 2019, 15, 1805087.

(7) Holá, K.; Marková, Z.; Zoppellaro, G.; Tuček, J.; Zbořil, R. Tailored Functionalization of 
Iron Oxide Nanoparticles for MRI, Drug Delivery, Magnetic Separation and Immobilization of Biosubstances. Biotechnol. Adv. 2015, 33, 1162-1176.

(8) Li, M.; Chen, T.; Gooding, J. J.; Liu, J. Review of Carbon and Graphene Quantum Dots for Sensing. ACS Sens. 2019, 4, 1732-1748.

(9) Xiao, L.; Sun, H. Novel Properties and Applications of Carbon Nanodots. Nanoscale Horiz.

2018, 3, 565-597.

(10) Zhu, S.; Song, Y.; Zhao, X.; Shao, J.; Zhang, J.; Yang, B. The Photoluminescence Mechanism in Carbon Dots (Graphene Quantum Dots, Carbon Nanodots, and Polymer Dots): Current State and Future Perspective. Nano Res. 2015, 8, 355-381.

(11) Wang, Y.; Hu, A. Carbon Quantum Dots: Synthesis, Properties and Applications. J. Mater. Chem. C 2014, 2, 6921-6939.

(12) Roy, P.; Chen, P. C.; Periasamy, A.P.; Chen, Y. N.; Chang, H. T. Photoluminescent Carbon Nanodots: Synthesis, Physicochemical Properties and Analytical Applications. Mater. Today 2015, 18, 447-458.

(13) Gharat, P. M.; Chethodil, J. M.; Srivastava, A. P.; Praseetha, P. K.; Pal, H.; Dutta Choudhury, S. An Insight into the Molecular and Surface State Photoluminescence of Carbon Dots Revealed through Solvent-Induced Modulations in Their Excitation Wavelength Dependent Emission Properties. Photochem. Photobiol. Sci. 2019, 18, 110119.

(14) Li, X.; Zhang, S.; Kulinich, S. A.; Liu, Y.; Zeng, H. Engineering Surface States of Carbon Dots to Achieve Controllable Luminescence for Solid-Luminescent Composites and 
Sensitive Be ${ }^{2+}$ Detection. Sci.Rep. 2014, 4, 4976.

(15) Ding, H.; Yu, S. B.; Wei, J. S.; Xiong, H. M. Full-Color Light-Emitting Carbon Dots with a Surface-State-Controlled Luminescence Mechanism. ACS Nano 2016, 10, 484-491.

(16) Shamsipur, M.; Barati, A.; Taherpour, A. A.; Jamshidi, M. Resolving the Multiple Emission Centers in Carbon Dots: From Fluorophore Molecular States to Aromatic Domain States and Carbon-Core States. J. Phys. Chem. Lett. 2018, 9, 4189-4198.

(17) Song, Y.; Zhu, S.; Zhang, S.; Fu, Y.; Wang, L.; Zhao, X.; Yang, B. Investigation from Chemical Structure to Photoluminescent Mechanism: A Type of Carbon Dots from the Pyrolysis of Citric Acid and an Amine. J. Mater. Chem. C 2015, 3, 5976-5984.

(18) Schneider, J.; Reckmeier, C. J.; Xiong, Y.; Von Seckendorff, M.; Susha, A. S.; Kasák, P.; Rogach, A. L. Molecular Fluorescence in Citric Acid-Based Carbon Dots. J. Phys. Chem. C 2017, 121, 2014-2022.

(19) Gude, V.; Das, A.; Chatterjee, T.; Mandal, P. K. Molecular Origin of Photoluminescence of Carbon Dots: Aggregation-Induced Orange-Red Emission. Phys. Chem. Chem. Phys. 2016, $18,28274-28280$.

(20) Liu, M. L.; Chen, B. B.; Li, C. M.; Huang, C. Z. Carbon Dots: Synthesis, Formation Mechanism, Fluorescence Origin and Sensing Applications. Green Chem. 2019, 21, 449471.

(21) Mintz, K. J.; Zhou, Y.; Leblanc, R. M. Recent Development of Carbon Quantum Dots Regarding Their Optical Properties, Photoluminescence Mechanism, and Core Structure. Nanoscale 2019, 11, 4634-4652. 
(22) Wang, T.; Wang, A.; Wang, R.; Liu, Z.; Sun, Y.; Shan, G.; Chen, Y.; Liu, Y. Carbon Dots with Molecular Fluorescence and Their Application as a "Turn-off" Fluorescent Probe for Ferricyanide Detection. Sci.Rep. 2019, 9, 10723.

Qu, D.; Sun, Z. The Formation Mechanism and Fluorophores of Carbon Dots Synthesized: Via a Bottom-up Route. Mater. Chem. Front. 2020, 4, 400-420.

(24) Ehrat, F.; Bhattacharyya, S.; Schneider, J.; Löf, A.; Wyrwich, R.; Rogach, A. L.; Stolarczyk, J. K.; Urban, A. S.; Feldmann, J. Tracking the Source of Carbon Dot Photoluminescence: Aromatic Domains versus Molecular Fluorophores. Nano Lett. 2017, 17, 7710-7716.

(25) Meierhofer, F.; Dissinger, F.; Weigert, F.; Jungclaus, J.; Müller-Caspary, K.; Waldvogel, S. R.; Resch-Genger, U.; Voss, T. Citric Acid Based Carbon Dots with Amine Type Stabilizers: pH-Specific Luminescence and Quantum Yield Characteristics. J. Phys. Chem. C 2020, 124, 8894-8904.

(26) Xiong, Y.; Schneider, J.; Ushakova, E. V.; Rogach, A. L. Influence of Molecular Fluorophores on the Research Field of Chemically Synthesized Carbon Dots. Nano Today 2018, 23, 124-139.

(27) Zhang, J.; Yang, L.; Yuan, Y.; Jiang, J.; Yu, S.-H. One-Pot Gram-Scale Synthesis of Nitrogen and Sulfur Embedded Organic Dots with Distinctive Fluorescence Behaviors in Free and Aggregated States. Chem. Mater. 2016, 28, 4367-4374.

(28) Shi, L.; Yang, J. H.; Zeng, H. B.; Chen, Y. M.; Yang, S. C.; Wu, C.; Zeng, H.; Yoshihito, O.; Zhang, Q. Carbon Dots with High Fluorescence Quantum Yield: The Fluorescence Originates from Organic Fluorophores. Nanoscale 2016, 8, 14374-14378. 
(29) Kasak, P.; Danko, M.; Zavahir, S.; Mrlik, M.; Xiong, Y.; Yousaf, A. B.; Lai, W.-F.; Krupa, I.; Tkac, J.; Rogach, A. L. Identification of Molecular Fluorophore as a Component of Carbon Dots Able to Induce Gelation in a Fluorescent Multivalent-Metal-Ion-Free Alginate Hydrogel. Sci.Rep. 2019, 9, 15080.

(30) Das, A.; Gude, V.; Roy, D.; Chatterjee, T.; De, C. K.; Mandal, P. K. On the Molecular Origin of Photoluminescence of Nonblinking Carbon Dot. J. Phys. Chem. C 2017, 121, 9634-9641.

(31) Reckmeier, C. J.; Schneider, J.; Xiong, Y.; Häusler, J.; Kasák, P.; Schnick, W.; Rogach, A. L. Aggregated Molecular Fluorophores in the Ammonothermal Synthesis of Carbon Dots. Chem. Mater. 2017, 29, 10352-10361.

(32) Mura, S.; Stagi, L.; Malfatti, L.; Carbonaro, C. M.; Ludmerczki, R.; Innocenzi, P. Modulating the Optical Properties of Citrazinic Acid through the Monomer-to-Dimer Transformation. J. Phys. Chem. A 2020, 124, 197-203.

(33) Chen, Z.; Stepanenko, V.; Dehm, V.; Prins, P.; Siebbeles, L. D. A.; Seibt, J.; Marquetand, P.; Engel, V.; Würthner, F. Photoluminescence and Conductivity of Self-Assembled $\pi-\pi$ Stacks of Perylene Bisimide Dyes. Chem. - Eur. J. 2007, 13, 436-449.

(34) Banal, J. L.; White, J. M.; Ghiggino, K. P.; Wong, W. W. H. Concentrating AggregationInduced Fluorescence in Planar Waveguides: A Proof-of-Principle. Sci. Rep. 2014, 4, 4635.

(35) Trouillas, P.; Sancho-García, J. C.; De Freitas, V.; Gierschner, J.; Otyepka, M.; Dangles, O. Stabilizing and Modulating Color by Copigmentation: Insights from Theory and Experiment. Chem. Rev. 2016, 116, 4937-4982. 
(36) Fu, M.; Ehrat, F.; Wang, Y.; Milowska, K. Z.; Reckmeier, C.; Rogach, A. L.; Stolarczyk, J. K.; Urban, A. S.; Feldmann, J. Carbon Dots: A Unique Fluorescent Cocktail of Polycyclic Aromatic Hydrocarbons. Nano Lett. 2015, 15, 6030-6035.

(37) Feng, J.; Dong, H.; Pang, B.; Shao, F.; Zhang, C.; Yu, L.; Dong, L. Theoretical Study on the Optical and Electronic Properties of Graphene Quantum Dots Doped with Heteroatoms. Phys. Chem. Chem. Phys. 2018, 20, 15244-15252.

(38) Sudolská, M.; Dubecký, M.; Sarkar, S.; Reckmeier, C. J.; Zbořil, R.; Rogach, A. L.; Otyepka, M. Nature of Absorption Bands in Oxygen-Functionalized Graphitic Carbon Dots. J. Phys. Chem. C 2015, 119, 13369-13373.

(39) Sudolská, M.; Otyepka, M. Exact Roles of Individual Chemical Forms of Nitrogen in the Photoluminescent Properties of Nitrogen-Doped Carbon Dots. Appl. Mater. Today 2017, 7 , 190-200.

(40) Wang, J.; Cao, S.; Ding, Y.; Ma, F.; Lu, W.; Sun, M. Theoretical Investigations of Optical Origins of Fluorescent Graphene Quantum Dots. Sci.Rep. 2016, 6, 24850.

(41) Yuan, F.; Yuan, T.; Sui, L.; Wang, Z.; Xi, Z.; Li, Y.; Li, X.; Fan, L.; Tan, Z.; Chen, A.; et al. Engineering Triangular Carbon Quantum Dots with Unprecedented Narrow Bandwidth Emission for Multicolored LEDs. Nat. Commun. 2018, 9, 2249.

(42) Carbonaro, C. M.; Chiriu, D.; Stagi, L.; Casula, M. F.; Thakkar, S. V.; Malfatti, L.; Suzuki, K.; Ricci, P. C.; Corpino, R. Carbon Dots in Water and Mesoporous Matrix: Chasing the Origin of Their Photoluminescence. J. Phys. Chem. C 2018, 122, 25638-25650.

(43) MarvinSketch 18.17.0, ChemAxon (https://www.chemaxon.com). 
(44) Paloncýová, M.; Langer, M.; Otyepka, M. Structural Dynamics of Carbon Dots in Water and $N, N$-Dimethylformamide Probed by All-Atom Molecular Dynamics Simulations. $J$. Chem. Theory Comput. 2018, 14, 2076-2083.

(45) Yang, H.; Liu, Y.; Guo, Z.; Lei, B.; Zhuang, J.; Zhang, X.; Liu, Z.; Hu, C. Hydrophobic Carbon Dots with Blue Dispersed Emission and Red Aggregation-Induced Emission. Nat. Commun. 2019, 10, 1789.

(46) Reckmeier, C. J.; Schneider, J.; Susha, A. S.; Rogach, A. L. Luminescent Colloidal Carbon Dots: Optical Properties and Effects of Doping [Invited]. Opt. Express 2016, 24, A312A340.

(47) Jeziorski, B.; Moszynski, R.; Szalewicz, K. Perturbation Theory Approach to Intermolecular Potential Energy Surfaces of van Der Waals Complexes. Chem. Rev. 1994, $94,1887-1930$.

(48) Lu, S.; Cong, R.; Zhu, S.; Zhao, X.; Liu, J.; Tse, J. S.; Meng, S.; Yang, B. pH-Dependent Synthesis of Novel Structure-Controllable Polymer-Carbon NanoDots with High Acidophilic Luminescence and Super Carbon Dots Assembly for White Light-Emitting Diodes. ACS Appl. Mater. Interfaces 2016, 8, 4062-4068.

(49) Russo, C.; Apicella, B.; Ciajolo, A. Blue and Green Luminescent Carbon Nanodots from Controllable Fuel-Rich Flame Reactors. Sci. Rep. 2019, 9, 14566.

(50) Baker, S. N.; Baker, G. A. Luminescent Carbon Nanodots: Emergent Nanolights. Angew. Chem., Int. Ed. 2010, 49, 6726-6744.

(51) Zhu, S.; Song, Y.; Wang, J.; Wan, H.; Zhang, Y.; Ning, Y.; Yang, B. Photoluminescence 
Mechanism in Graphene Quantum Dots: Quantum Confinement Effect and Surface/Edge State. Nano Today 2017, 13, 10-14.

(52) Zhou, J.; Booker, C.; Li, R.; Zhou, X.; Sham, T.-K.; Sun, X.; Ding, Z. An Electrochemical Avenue to Blue Luminescent Nanocrystals from Multiwalled Carbon Nanotubes (MWCNTs). J. Am. Chem. Soc. 2007, 129, 744-745.

(53) Luzar, A.; Chandler, D. Hydrogen-Bond Kinetics in Liquid Water. Nature 1996, 379, 5557.

(54) Luzar, A. Resolving the Hydrogen Bond Dynamics Conundrum. J. Chem. Phys. 2000, 113, 10663-10675.

(55) Wang, J.; Wolf, R. M.; Caldwell, J. W.; Kollman, P. A.; Case, D. A. Development and Testing of a General Amber Force Field. J. Comput. Chem. 2004, 25, 1157-1174.

Siddique, F.; Langer, M.; Paloncýová, M.; Medved', M.; Otyepka, M.; Nachtigallová, D.; Lischka, H.; Aquino, A. J. A. Conformational Behavior and Optical Properties of a Fluorophore Dimer as Model of Luminescent Centers in Carbon Dots. J. Phys. Chem. C 2020, $124,14327-14337$.

(57) Wang, J.; Cieplak, P.; Kollman, P. A. How Well Does a Restrained Electrostatic Potential (RESP) Model Perform in Calculating Conformational Energies of Organic and Biological Molecules? J. Comput. Chem. 2000, 21, 1049-1074.

(58) Cheng, A.; Steele, W. A. Computer Simulation of Ammonia on Graphite. I. Low Temperature Structure of Monolayer and Bilayer Films. J. Chem. Phys. 1990, 92, 38583866. 
(59) Berendsen, H. J. C.; Grigera, J. R.; Straatsma, T. P. The Missing Term in Effective Pair Potentials. J. Phys. Chem. 1987, 91, 6269-6271.

(60) Ȧqvist, J. Ion-Water Interaction Potentials Derived from Free Energy Perturbation Simulations. J. Phys. Chem. 1990, 94, 8021-8024.

(61) Hess, B.; Bekker, H.; Berendsen, H. J. C.; Fraaije, J. G. E. M. LINCS: A Linear Constraint Solver for Molecular Simulations. J. Comput. Chem. 1997, 18, 1463-1472.

(62) Bussi, G.; Donadio, D.; Parrinello, M. Canonical Sampling Through Velocity Rescaling. J. Chem. Phys. 2007, 126, 014101.

(63) Berendsen, H. J. C.; Postma, J. P. M.; van Gunsteren, W. F.; DiNola, A.; Haak, J. R. Molecular Dynamics with Coupling to an External Bath. J. Chem. Phys. 1984, 81, 36843690.

(64) Darden, T.; York, D.; Pedersen, L. Particle Mesh Ewald: An N·Log(N) Method for Ewald Sums in Large Systems. J. Chem. Phys. 1993, 98, 10089-10092.

(65) Van Der Spoel, D.; Lindahl, E.; Hess, B.; Groenhof, G.; Mark, A. E.; Berendsen, H. J. C. GROMACS: Fast, Flexible, and Free. J. Comput. Chem. 2005, 26, 1701-1718.

(66) Mlýnský, V.; Kührová, P.; Kühr, T.; Otyepka, M.; Bussi, G.; Banáš, P.; Šponer, J. FineTuning of the AMBER RNA Force Field with a New Term Adjusting Interactions of Terminal Nucleotides. J. Chem. Theory Comput. 2020, 16, 3936-3946.

(67) The PyMOL Molecular Graphics System, Version 1.8. Schrodinger, LLC. 